\title{
EF-hand like Region in the N-terminus of Anoctamin 1 Modulates Channel Activity by $\mathrm{Ca}^{2+}$ and Voltage
}

\author{
Min Ho Tak ${ }^{1}$, Yongwoo Jang ${ }^{2}$, Woo Sung Son ${ }^{3}$, Young Duk Yang ${ }^{3 *}$ and Uhtaek Oh ${ }^{1,4 *}$ \\ ${ }^{1}$ Interdisciplinary Program in Neuroscience, Seoul National University, Seoul 08826, ${ }^{2}$ Department of Biomedical Engineering, \\ Hanyang University, Seoul 04763, ${ }^{3}$ College of Pharmacy, CHA University, Seongnam 13488, ${ }^{4}$ Sensory Research Center, Brain \\ Science Institute, Korea Institute of Science \& Technology (KIST), Seoul 02792, Korea
}

\begin{abstract}
Anoctamin 1 (ANO1) also known as TMEM16A is a transmembrane protein that functions as a $\mathrm{Ca}^{2+}$ activated chloride channel. Recently, the structure determination of a fungal Nectria haematococca TMEM16 (nhTMEM16) scramblase by X-ray crystallography and a mouse ANO1 by cryo-electron microscopy has provided the insight in molecular architecture underlying phospholipid scrambling and $\mathrm{Ca}^{2+}$ binding. Because the $\mathrm{Ca}^{2+}$ binding motif is embedded inside channel protein according to defined structure, it is still unclear how intracellular $\mathrm{Ca}^{2+}$ moves to its deep binding pocket effectively. Here we show that EF-hand like region containing multiple acidic amino acids at the N-terminus of ANO1 is a putative site regulating the activity of ANO1 by $\mathrm{Ca}^{2+}$ and voltage. The EF-hand like region of ANO1 is highly homologous to the canonical EF hand loop in calmodulin that contains acidic residues in key $\mathrm{Ca}^{2+}$-coordinating positions in the canonical EF hand. Indeed, deletion and Ala-substituted mutation of this region resulted in a significant reduction in the response to $\mathrm{Ca}^{2+}$ and changes in its key biophysical properties evoked by voltage pulses. Furthermore, only ANO1 and ANO2, and not the other TMEM16 isoforms, contain the EF-hand like region and are activated by Ca ${ }^{2+}$. Moreover, the molecular modeling analysis supports that EF-hand like region could play a key role during $\mathrm{Ca}^{2+}$ transfer. Therefore, these findings suggest that EF-hand like region in ANO1 coordinates with $\mathrm{Ca}^{2+}$ and modulate the activation by $\mathrm{Ca}^{2+}$ and voltage.
\end{abstract}

Key words: Anoctamin-1, Chloride channels, Calcium, Mutagenesis, Site-directed

\section{INTRODUCTION}

Endogenous $\mathrm{Ca}^{2+}$-activated chloride channels (CaCCs) regulate anionic currents by intracellular $\mathrm{Ca}^{2+}$ as well as by voltage [1-4]. Although CaCCs mediate diverse functions in various tissues, their biophysical properties display remarkable homogeneity. Their typical properties can be summarized as; activation by intracellular $\mathrm{Ca}^{2+}$ and voltage, an outwardly rectifying current-voltage relationship, slow time-dependent activation by voltage pulses,

Received May 23, 2019, Revised November 30, 2019 ,

Accepted December 11,2019

* To whom correspondence should be addressed.

Young Duk Yang, TEL: 82-31-881-7170, FAX: 82-31-881-7219

e-mail:ntsky0816@cha.ac.kr

Uhtaek Oh, TEL: 82-2-958-7031, FAX: 82-2-958-7034

e-mail:utoh@kist.re.kr and voltage-dependent slow decay of tail currents [2]. Anoctamin 1 (ANO1) cloned for a CaCC has the biophysical and the pharmacological properties of native CaCCs [5-7]. For example, it is activated by $\mathrm{Ca}^{2+}$ - and voltage, blocked by $\mathrm{CaCC}$ inhibitors, stimulated by G-protein-coupled receptor ligands via the $\mathrm{PLC} / \mathrm{IP}_{3}$ pathway, expressed in transport epithelia, and has an outwardly rectifying $\mathrm{I} \sim \mathrm{V}$ relationship [5-7]. ANO1 has four different splice variants, and of these, splice variants with exon $6 \mathrm{~b}$ or exon 13 are known to affect $\mathrm{Ca}^{2+}$ sensitivity [8].

Because CaCCs are essential for mediating various physiological functions, an understanding of their activation mechanisms induced by intracellular $\mathrm{Ca}^{2+}$ and voltage would provide important insight of their functions. Several studies proposed the gating mechanism and putative binding residues by intracellular $\mathrm{Ca}^{2+}$. For example, Xiao and colleagues report that a poly-glutamate region in the first intracellular loop is important for coupling the volt-
Copyright (C) Experimental Neurobiology 2019.

www.enjournal.org
This is an Open Access article distributed under the terms of the Creative Commons Attribution Non-Commercial License (http://creativecommons.org/licenses/by-nc/4.0) which permits unrestricted non-commercial use, distribution, and reproduction in any medium, provided the original work is properly cited. 
age- and $\mathrm{Ca}^{2+}$-dependent gating of ANO1 [9]. Moreover, Lee and colleagues suggested that two helices in the third intracellular loop coordinate a conformational change in a $\mathrm{Ca}^{2+}$-dependent pushand-pull fashion [10]. Based on a positively charged ion of $\mathrm{Ca}^{2+}$, Tien and colleagues conducted systemic mutagenesis of all conserved acidic residues of ANO1, and concluded four putative amino acids (E650, E698, E701, and E730) are critical for $\mathrm{Ca}^{2+}$-induced ANO1 activation [11]. Recently, the crystal structure of the fungus Nectria haematococca TMEM16 (nhTMEM16) has been identified [12]. Based on the crystal structure, a calcium binding site has been proposed. The nhTMEM16 structure reveals that a putative $\mathrm{Ca}^{2+}$ binding site within subunit cavity is positioned by acidic amino acid residues in alpha-helices 6 and 7. The $\mathrm{Ca}^{2+}$-dependent lipid scrambling is disappeared by point mutations on several residues on glutamate and aspartate. More recently, mouse ANO1's structure further characterized by cryo-electron microscopy (cryo-EM) in 3 4 A resolution, which turned out a hydrophilic membraneexposed cavity that is a path for lipids in scramblases has changed to form an enclosed aqueous pore in a TMEM16 channel [13].

Although the $\mathrm{Ca}^{2+}$ binding site was determined in an nhTMEM16, the action mechanism underlying the activation of mammalian ANO1 and $\mathrm{ANO} 2$ by $\mathrm{Ca}^{2+}$ has still remained unclear. Based on the crystal structure of an nhTMEM16, the $\mathrm{Ca}^{2+}$ binding motif is deeply embedded inside phospholipid bilayer. Therefore, it is assumed the modulatory residues to help the movement of $\mathrm{Ca}^{2+}$ ions to the inside $\mathrm{Ca}^{2+}$ binding pocket of ANO1. In the present study, we set out to find the important residues to modulate channel activation of mouse ANO1. Especially, we investigated the role of the EF-hand like region in the N-terminus of ANO1 in both $\mathrm{Ca}^{2+}$ - and voltage- dependent ANO1 activation.

\section{MATERIALS AND METHODS}

\section{Mutagenesis and gene expression}

All mutants were generated from the wild type construct, mouse ANO1 (pEGFP-N1-mANO1). Amino-acid substitution or deletion mutants were made using a site-directed mutagenesis kit (Muta-direct, iNtRON Biotech, Seoul, Korea) or by the overlapPCR method. Mutations in all mutants were confirmed by sequencing whole nucleotide sequences.

HEK 293T cells were transfected with $1 \mu \mathrm{g}$ of pEGFP-N1-mANO1 or pEGFP-N1-mutants, $0.05 \mu \mathrm{g}$ pEGFP-N1, and FuGENE (Roche Diagnostics, Penzberg, Germany) in 35-mm Petri dishes. Transfected cells were incubated in DMEM supplemented with $10 \%$ fetal bovine serum (GIBCO) and penicillin-streptomycin at $37^{\circ} \mathrm{C}$ in a $5 \% \mathrm{CO}_{2}$ incubator. Cells were used one or two days after plating.

\section{Electrophysiology}

As soon as a borosilicate glass pipette (World Precision Instruments, Sarasota, FL) touched the surface of a HEK cell, a gentle suction was applied to the pipette to obtain gigaseal. The tip resistance of the pipette was about 3 Mohms for whole-cell recordings. To record whole-cell currents, the membrane in contact with the pipette was ruptured by applying gentle suction after forming gigaseals. Once a whole cell was formed, the capacitive transients were canceled. Currents were recorded with a patch-clamp amplifier (Axopatch 200B, Molecular Devices) and filtered at $5 \mathrm{KHz}$. The whole-cell currents activated by each $\mathrm{Ca}^{2+}$ concentration (0.1 100 uM) were recorded from a holding potential of $-60 \mathrm{mV}$ during voltage clamp steps from -100 to $+100 \mathrm{mV}$ by $20 \mathrm{mV}$ increments with $765 \mathrm{~ms}$ (-60 mV: $65 \mathrm{~ms},-100 \sim 100 \mathrm{mV}: 500 \mathrm{~ms}$, $-120 \mathrm{mV}: 200 \mathrm{~ms}$ ) total duration. Data from the amplifier were digitized with Digidata 1440A (Molecular Devices) and stored in a personal computer.

ANO1 currents were activated by intracellular $\mathrm{Ca}^{2+}$ at a holding potential of - $60 \mathrm{mV}$ unless specified. For recording currents in response to voltage pulses, membrane potential was changed from $-100 \mathrm{mV}$ to $+100 \mathrm{mV}$ in $20 \mathrm{mV}$ increment. Square voltage pulses of $500 \mathrm{~ms}$ duration were delivered from a holding potential of -60 $\mathrm{mV}$. Tail currents were recorded at $-120 \mathrm{mV}$.

The control pipette solution contained $140 \mathrm{mM}$ NMDG-Cl, 2 $\mathrm{mM} \mathrm{MgCl}_{2}$, and $10 \mathrm{mM}$ HEPES adjusted to $\mathrm{pH}$ 7.2. For the $\mathrm{Ca}^{2+}-$ free solution, $1 \mathrm{mM}$ EGTA was added to the control solution.

To calculate the accurate free intracellular $\mathrm{Ca}^{2+}$ concentration $\left(\left[\mathrm{Ca}^{2+}\right]_{\mathrm{i}}\right)$ in pipette solutions, the WEBMAXC program in website (http://www.stanford.edu/ cpatton/webmaxc/webmaxcS.htm) was used. The bath solution contained $140 \mathrm{mM}$ NMDG-Cl, $2 \mathrm{mM}$ $\mathrm{MgCl}_{2}$, and $10 \mathrm{mM} \mathrm{HEPES}$ and was adjusted to $\mathrm{pH}$ 7.2.

Whole cell peak currents were recorded at each free $\left[\mathrm{Ca}^{2+}\right]_{i}$, and then averaged for subsequent analysis. Half-maximal concentrations $\left(\mathrm{EC}_{50}\right)$ were calculated using averaged peak currents, and fitted using the Hill equation. Deactivation time constants $\left(\tau_{\mathrm{d}}\right)$ were derived by the single exponential fitting of tail currents recorded at $-120 \mathrm{mV}$ for $150 \mathrm{~ms}$.

\section{Molecular modeling of ANO1}

To construct three-dimensional structure of mouse ANO1 using in silico method, the crystal structure of TMEM16 from the fungus Nectria haematococca (PDB ID:4WIS, nhTMEM16) was used [12]. Using I-TASSER [14] algorithm, the modeled structures were generated with template structure (4WIS), and energy minimization of structures were performed with the Amber force field $[15,16]$ with UCSF Chimera [17]. During energy minimization, AMBER force field parameters were set to the basic values 
in UCSF Chimera and Antechamber module was used to adjust parameters to non-standard residues. In energy minimization step, steepest descent (100 steps) and conjugate gradient (100 steps) minimization were used to relieve unfavorable clashed region. Size of all minimization steps was $0.02 \AA$. Structural alignment of modeled structure and nhTMEM16 was performed using MatchMaker modules in UCSF Chimera. During structural alignment, Needleman-Wunsch alignment algorithm was used with Blosum-62 matrix and 30\% secondary structure score [18]. Structural matching process was iterated by pruning long atom pairs until no pair exceeds of 2.0 angstroms. For structure refinement analysis in water solvent, the adaptive Poisson-Boltzmann software (APBS) [19] and Coulombic surface coloring method in UCSF Chimera were used to analyze the electrostatic surface potential (ESP) of modeled structures. The electrostatic surface potential maps of structures were generated by numerically solving the Poisson-Boltzmann equation based on molecular mechanics. Structure validation was performed with Protein Structure Validation Software Suite (PSVS) [20]. Molecular visualization of structure was done using UCSF Chimera. Also, Probis algorithm was used to predict potent $\mathrm{Ca}^{2+}$ modulating site of ANO1 [21].

\section{RESULTS}

\section{Mutation in the EF-hand like region of ANO1 changes acti- vation kinetics evoked by $\mathrm{Ca}^{2+}$}

In mammals, calmodulin is a multifunctional intermediate protein that is regulated by $\mathrm{Ca}^{2+}$. The intracellular $\mathrm{Ca}^{2+}$ ions have well known to directly bind to EF-hand motif that is an signature sequence of the most common (canonical) EF-hand (-DxDxDGxxxxxE-) of calmodulin [22, 23]. Interestingly, we first found that the amino acid residues (285-DGDYEGDNVE-294) in the $\mathrm{N}$-terminus of ANO1 are somewhat similar to the EF-hand motif of calmodulin (Fig. 1A). Thus, we designated this region as the EF-hand like region in the present study. To investigate the effect of EF-hand like region on the activity of ANO1, we constructed ANO1 mutants in the region and then compared their activity by $\mathrm{Ca}^{2+}$ and voltage with those of the wild-type.

After forming whole cells with $10 \mu \mathrm{M} \mathrm{Ca}^{2+}$ in the pipette, large, robust currents were evoked in HEK cells transfected with wildtype ANO1. However, whole-cell currents to $\mathrm{Ca}^{2+}$ desensitized rapidly (Fig. 1B and 1C). In contrast to wild-type ANO1, whole-cell currents of $\Delta 285 \sim 297$ mutant to $10 \mu \mathrm{M} \mathrm{Ca}^{2+}$ were barely detectable (Fig. 1B and 1C). Therefore, Ala substitution was performed to determine the important amino acids in this region. More specifically, negatively charged amino acids, Asp or Glu at positions $285,287,289$, or 291 were replaced by Ala. The Ala-substitution analysis revealed that D287A and D291A mutants elicited current responses comparable to that of wild type ANO1. However, the D285A and E289A mutants showed significant reductions in current response (Fig. 1B and 1C). The double (D285A/D287A) and triple Ala (D285A/D287A/E289A, 3A) substitution mutants also showed significant reductions in current density to $10 \mu \mathrm{M}$ $\mathrm{Ca}^{2+}$ (Fig. 1B and 1C). When four or five acidic amino acids in 285-DGDYEGDNVE-294 were replaced with Ala, the mutants AGAYAGA (4A) and AGAYAGANVA (5A) showed marked reductions in current response to $10 \mu \mathrm{M} \mathrm{Ca}^{2+}$ (Fig. $1 \mathrm{~B}$ and $1 \mathrm{C}$ ), suggesting that all five acidic amino acids in this region contribute to activation of ANO1 by $\mathrm{Ca}^{2+}$.

To further investigate the responsiveness to $\mathrm{Ca}^{2+}$, different concentrations of $\mathrm{Ca}^{2+}$ were applied to the bath of inside-out membrane patches to investigate dose-response relationships. Because single-channel currents of ANO1 mutants activated by $\mathrm{Ca}^{2+}$ were too small to measure at $-60 \mathrm{mV}$, the holding potential was set at $+80 \mathrm{mV}$ in order to increase current amplitudes. When the concentration of $\mathrm{Ca}^{2+}$ was increased from $0.01 \mu \mathrm{M}$ to $10 \mu \mathrm{M}$, macroscopic currents were observed to increase dose-dependently (Fig. 1D and 1E). The effective concentration $\left(\mathrm{EC}_{50}\right)$ of $\mathrm{Ca}^{2+}$ for the activation of wild-type ANO1 was $1.0 \mu \mathrm{M}(\mathrm{n}=5 \sim 11)$. Endogenous CaCCs are known to have a greater sensitivity to $\mathrm{Ca}^{2+}$ at depolarization [2-4]. Likewise, ANO1 also shows a slight increase in sensitivity to $\mathrm{Ca}^{2+}$ at depolarization. At $-80 \mathrm{mV}$, the $\mathrm{EC}_{50}$ of $\mathrm{ANO} 1$ was $1.4 \mu \mathrm{M}(\mathrm{n}=5 \sim 12)$. Furthermore, the macroscopic channel currents of ANO1 increased abruptly between 0.3 and $3 \mu \mathrm{M}$. Thus, the Hill coefficient of wild-type ANO1 was found to be 3.7, suggesting a high level of cooperativity among $\mathrm{Ca}^{2+}$ coordination sites. In contrast, magnitudes of macroscopic currents of AGAYAGA mutant activated by $\mathrm{Ca}^{2+}$ were much smaller than those of the wild-type (Fig. 1D and 1E). Furthermore, the dose-response curve of this mutant was markedly shifted rightward with a 35 -fold increase in $\mathrm{EC}_{50}(41.8 \mu \mathrm{M}, \mathrm{n}=6 \sim 16)$ versus wild-type ANO1 (Fig. 1D and 1E). Interestingly, the Hill-coefficient of the mutant was markedly lower at 0.6 , suggesting a lack of cooperativity among $\mathrm{Ca}^{2+}$ coordinating sites. Similarly, when two acidic amino acids in the EF-hand like region were replaced with $\mathrm{Ala}(\mathrm{D} 285 \mathrm{~A} / \mathrm{D} 287 \mathrm{~A})$, the $\mathrm{EC}_{50}$ was $5.8 \mu \mathrm{M}$, which represented a much smaller rightward shift than that observed for the AGAYAGA mutant. The Hill-coefficient of the D285A/D287A mutant was 0.9, again lower than that of the wild-type ANO1. It is possible that these mutations could alter protein stability or cell surface expression, which would result in impaired channel activity. Thus, to confirm the cell surface expression and protein stability of mutants, we constructed GFP-tagged wild type ANO1 and mutant ANO1 proteins. Transfection of these plasmids into HEK cells showed normal localization to the 


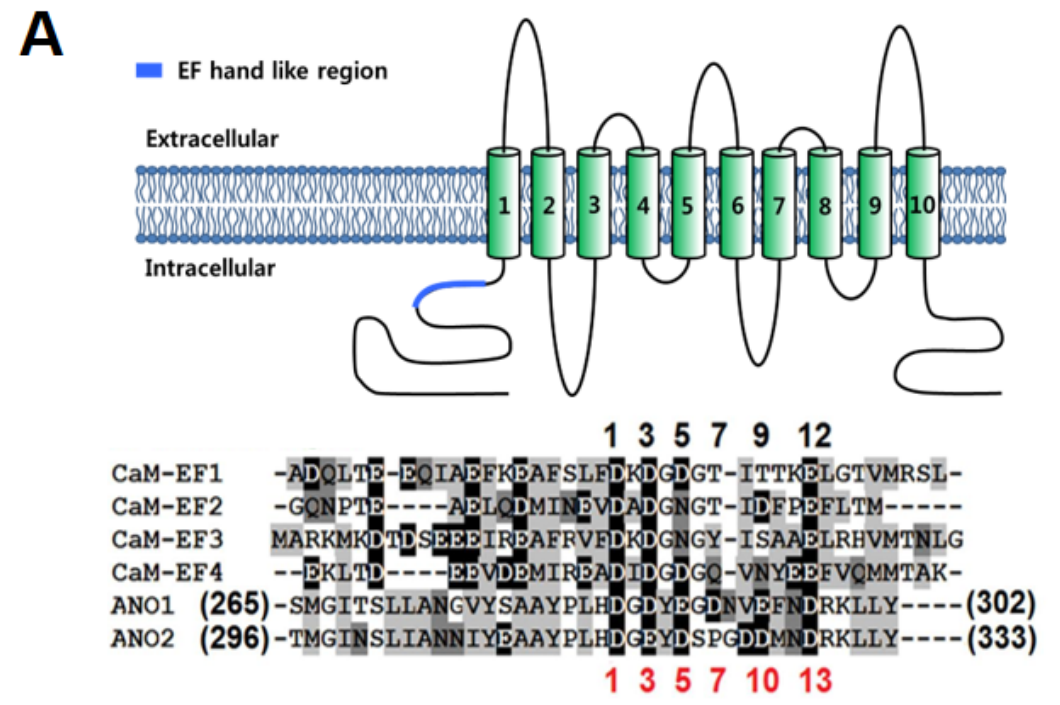

B

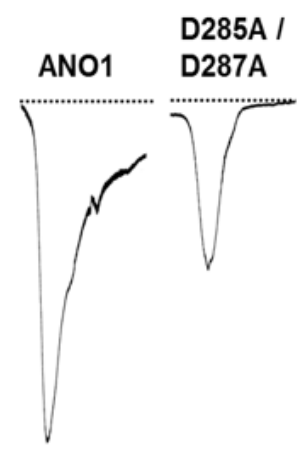

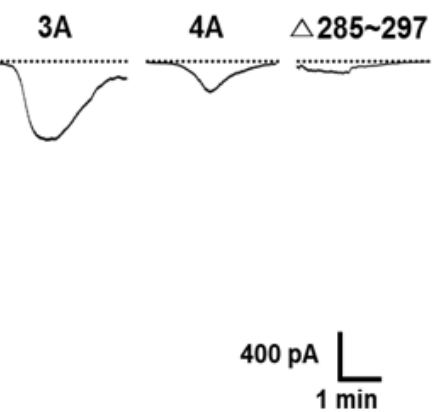

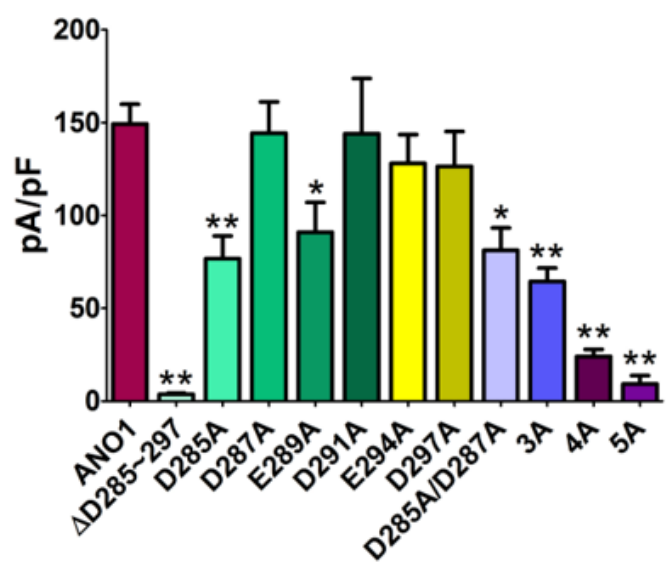

Fig. 1. Mutation of the EF-hand like region of ANO1 alters $\mathrm{Ca}^{2+}$ sensitivity. (A) Putative $\mathrm{Ca}^{2+}$ modulation sites in $\mathrm{ANO} 1$ and $\mathrm{ANO}$ : the EF-hand like region in the $\mathrm{N}$-terminus. The sequence alignment displays similarity between EF-hand loops of calmodulin and the EF-hand like regions in ANO1 and ANO2. (B) Whole cell currents of ANO1 and EF-hand like region mutants activated by $\left[\mathrm{Ca}^{2+}\right]_{\mathrm{i}}$ in HEK cells transfected with ANO1 and mutant genes. Whole-cell currents were elicited using a $10 \mu \mathrm{M} \mathrm{Ca}^{2+}$ pipette solution at a holding potential of $-60 \mathrm{mV}$. Pipette and bath solutions contained $140 \mathrm{mM}$ NMDG-Cl. The dashed line represents the baseline. 3A; D285A/D287A/E289A mutant, 4A; D285A/D287A/E289A/D291A (AGAYAGA) mutant. (C) Summarized whole cell currents of ANO1 and EF-hand like region mutants $(\mathrm{n}=5 \sim 16) .{ }^{* *} \mathrm{p}<0.01$ compared to ANO1 (ANOVA, Tukey's post-hoc test). Error bars represent SEMs. 5A; D285A/D287A/E289A/D291A/E294A mutant. (D) Representative traces of single-channel currents of wild-type ANO1 and its mutants activated by various concentrations of $\mathrm{Ca}^{2+} . \mathrm{Ca}^{2+}$ was applied to the bath of inside-out patches isolated from $\mathrm{HEK}$ cells expressing ANO1 and its 2A (D285A /D287A) or 4A mutant at different concentrations. The holding potential was $+80 \mathrm{mV}$. (E) Dose-response relationship of ANO1 (•) and of its $2 \mathrm{~A}(\boldsymbol{\nabla})$ and $4 \mathrm{~A}(\mathbf{-})$ EF-hand like region mutants. Plots of single-channel currents at each $\left[\mathrm{Ca}^{2+}\right]_{\mathrm{i}}$ concentration (from 0.01 to $\left.1,000 \mu \mathrm{M}\right)$ were fitted to Hill's equation $(\mathrm{n}=5 \sim 12)$. $\mathrm{E}_{\text {hold }}=+80 \mathrm{mV}$. ANO1, $\mathrm{EC}_{50}=1.0 \mu \mathrm{M}$ (Hill's coefficient $\left.\left(\mathrm{n}_{\mathrm{h}}\right)=3.7\right) ; 2 \mathrm{~A}, \mathrm{EC}_{50}=5.8 \mu \mathrm{M}\left(\mathrm{n}_{\mathrm{h}}=0.9\right) ; 4 \mathrm{~A}, \mathrm{EC}_{50}=41.8 \mu \mathrm{M}\left(\mathrm{n}_{\mathrm{h}}=0.6\right.$ ). (F) Localization of wild type ANO1 and mutants in HEK cells. GFP-tagged wild type ANO1, 4A and $\triangle 285-297$ mutants are normally observed in the plasma membrane. $(\mathrm{G})$ Western blot analysis of mutant ANO1s. HEK cells transfected with wild type ANO1 were used as positive control.

plasma membrane and protein expression level in both wild type and mutants (Fig. $1 \mathrm{~F}$ and $1 \mathrm{G}$ ). These results suggest that acidic amino acids in the EF-hand like region of ANO1 are essential for the activation by $\mathrm{Ca}^{2+}$. Furthermore, these results also suggest that the EF-hand like region of ANO1 will serve to transfer $\mathrm{Ca}^{2+}$ to the $\mathrm{Ca}^{2+}$ binding site through multiple interactions with $\mathrm{Ca}^{2+}$.

\section{Mutation in the EF-hand like region of ANO1 changes acti- vation kinetics evoked by voltage}

Voltage is also required for the activation of ANO1 [5]. However, the location in ANO1 responsible for its reaction to voltage pulses is not known. To examine ANO1 responses to voltage, voltage pulses from $-100 \mathrm{mV}$ to $100 \mathrm{mV}$ were applied in $20 \mathrm{mV}$ 
D

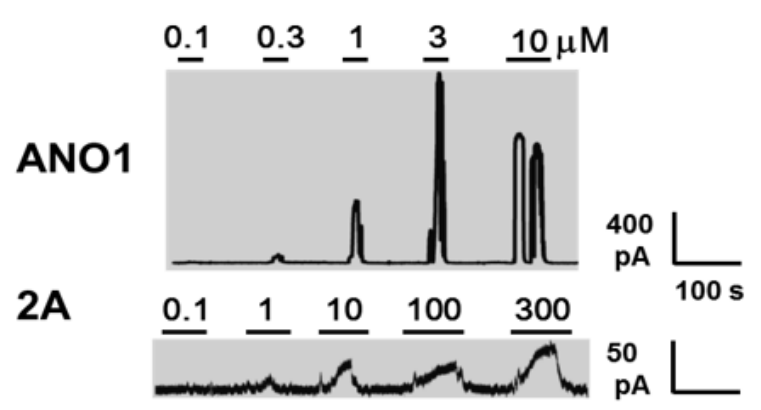

4A

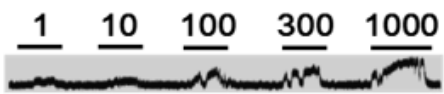

$\mathbf{F}$
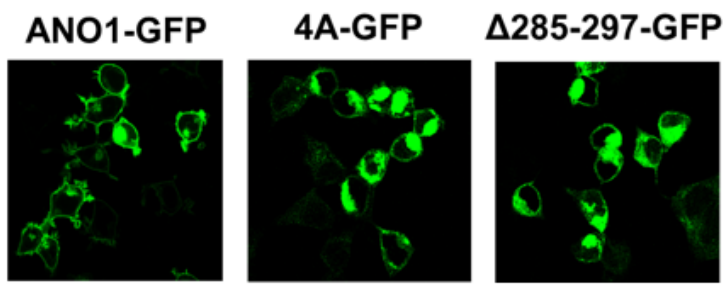

Fig. 1. Continued

increments to HEK cells expressing the wild-type or AGAYAGA mutant. As was observed for endogenous CaCCs [2,3], ANO1 was rarely activated by voltage with no $\mathrm{Ca}^{2+}$ in the pipette (no added $\mathrm{Ca}^{2+}$ and $5 \mathrm{mM}$ EGTA), but it was activated by voltage pulses when the intracellular $\mathrm{Ca}^{2+}$ concentration $\left(\left[\mathrm{Ca}^{2+}\right]_{\mathrm{i}}\right)$ was increased from 0.1 to $10 \mu \mathrm{M}$ (Fig. 2A). Furthermore, as $\left[\mathrm{Ca}^{2+}\right]_{i}$ was increased, current responses of wild-type ANO1 to voltage steps also increased (Fig. 2A). At depolarization, instantaneous ANO1 activation was followed by a slow activation that achieved peak current after a few hundred milliseconds (Fig. 2A). However, this slow activation was not observed at voltage pulses of less than $0 \mathrm{mV}$. Furthermore, the typical slow activation induced by depolarization became less time-dependent when $\left[\mathrm{Ca}^{2+}\right]_{i}$ was increased. Currents activated by voltage pulses were outwardly rectifying because ANO1 elicited much greater currents at depolarization than at hyperpolarization (Fig. 2B). Thus, ANO1 conductances were greater at depolarization (Fig. 2C). Similarly, ANO1 conductance was also found to be $\mathrm{Ca}^{2+}$ dependent; and conductance at each voltage increased as $\left[\mathrm{Ca}^{2+}\right]_{\mathrm{i}}$ was increased from 0.1 to $10 \mu \mathrm{M}$ (Fig. $2 \mathrm{C}$ ).

To investigate the kinetics of deactivation, tail currents activated by $-120 \mathrm{mV}$ after various pre-pulses were measured at different
$\mathbf{E}$

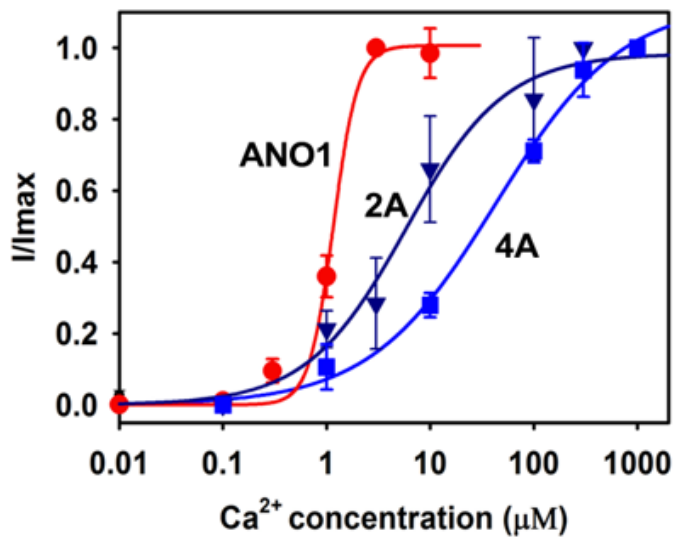

G

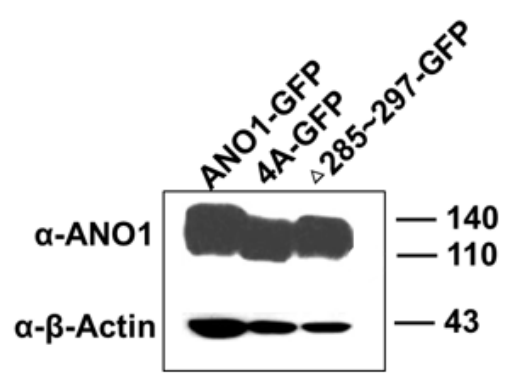

$\left[\mathrm{Ca}^{2+}\right]_{\mathrm{i}}$. As was observed for the tail currents of endogenous CaCCs $[2,4,24]$, ANO1 tail currents decayed slowly as the pre-pulse depolarized (Fig. 2A and 2D), which suggests the voltage-dependent deactivation of ANO1. Furthermore, tail current decay was also found to be $\mathrm{Ca}^{2+}$ dependent, as tail currents decayed more slowly on increasing $\left[\mathrm{Ca}^{2+}\right]_{i}$ (Fig. 2D). The deactivation time constant $\left(\tau_{\mathrm{d}}\right)$ was well fitted by a single exponential. $\tau_{\mathrm{d}}$ increased as $\left[\mathrm{Ca}^{2+}\right]_{\mathrm{i}}$ increased, especially at $\left[\mathrm{Ca}^{2+}\right]_{\mathrm{i}}>1 \mu \mathrm{M}$. But at low $\left[\mathrm{Ca}^{2+}\right]_{\mathrm{i}}(<0.3 \mu \mathrm{M}), \tau_{\mathrm{d}}$ did not change appreciably as voltage was changed. Furthermore, hyperpolarization $(-100 \mathrm{mV})$ deactivated ANO1 rapidly even in the presence of high $\left[\mathrm{Ca}^{2+}\right]_{\mathrm{i}}(10 \mu \mathrm{M})$ (Fig. 2A and 2D).

In contrast, the AGAYAGA mutant responded to voltage pulses differently from the wild-type. More specifically, this mutant showed much lower current responses to voltage pulses (Fig. 2A), and its I V curves at different $\left[\mathrm{Ca}^{2+}\right]_{i}$ were close to linear (Fig. 2B). Furthermore, the conductance of AGAYAGA was less dependent on voltage (Fig. 2C). In addition, its activation and deactivation kinetics were quite different from those of wild type ANO1. The characteristic slow activation observed at the depolarizing voltage was almost absent in the AGAYAGA mutant (Fig. 2A). Thus, its activation by voltage appeared time-independent. Similarly, unlike 

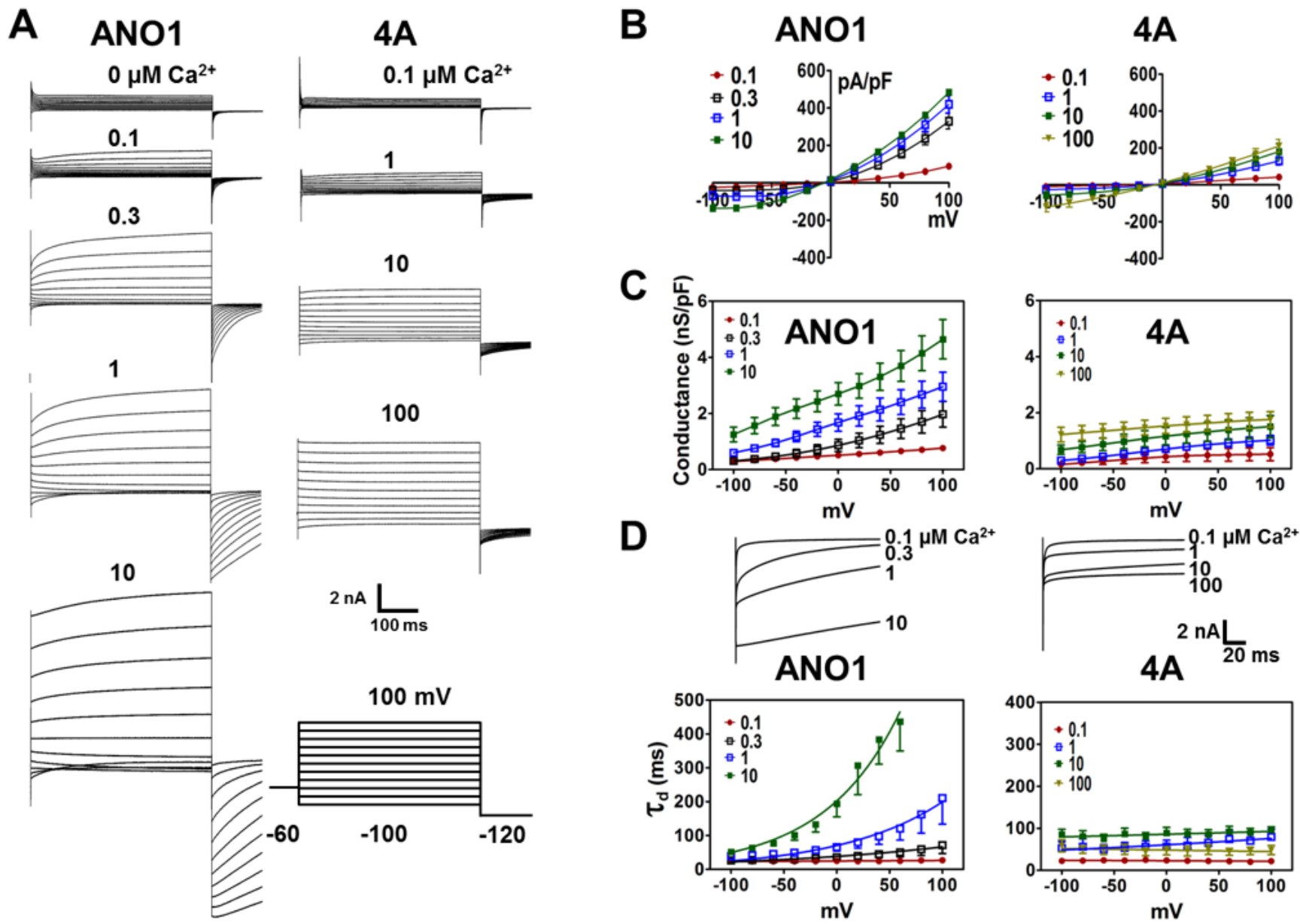

Fig. 2. Mutation in the EF-hand like region alters ANO1 characteristic responses to voltage steps. (A) Slow activation of outward currents at depolarization by low $\left[\mathrm{Ca}^{2+}\right]_{\mathrm{i}}$ and slow-decaying tail currents of ANO1 at high $\left[\mathrm{Ca}^{2+}\right]_{\mathrm{i}}$ (left panel) were almost absent in the $4 \mathrm{~A}$ mutant (right panel). Wholecell currents of ANO1 and its 4A mutant were recorded using a step voltage protocol (lower panel, from - 100 to $100 \mathrm{mV}$ in $20 \mathrm{mV}$ increments, $500 \mathrm{~ms}$ per step) at $\left[\mathrm{Ca}^{2+}\right]_{\mathrm{i}}$ levels of from 0 to $10 \mu \mathrm{M}$ for $\mathrm{ANO} 1$ and from 0.1 to $100 \mu \mathrm{M}$ for $4 \mathrm{~A}$ mutant. Tail currents were recorded at $-120 \mathrm{mV}$ over $150 \mathrm{~ms}$. (B) Current-voltage relationships of ANO1 (left panel) and 4A mutant (right panel). Data were taken from currents at the end of each voltage step (n=5). (C) The conductance-voltage relationships of tail currents $(n=5)$. Conductances were determined by measuring instantaneous tail currents at $-120 \mathrm{mV}$ after various voltage pulses. (D) The upper panel displays average tail currents of ANO1 (left panel) and 4A mutant (right panel) ( $\mathrm{n}=5 \sim 6$ ). The lower panel displays deactivation time constants obtained from the tail currents shown in the upper panel. Deactivation time constants $\left(\tau_{\mathrm{d}}\right)$ were obtained using single exponential fits to tail currents recorded at $-120 \mathrm{mV}$.

the wild-type, its deactivation was largely voltage independent at all $\left[\mathrm{Ca}^{2+}\right]_{i}$ examined, and $\tau_{d}$ was almost constant throughout the voltage pulse range (Fig. 2D). Thus, the EF-hand like region of ANO1 appears to contribute substantially to its voltage dependence.

\section{The EF-hand like region in ANO2}

Of the 10 known isoforms of the anoctamin channel family, $\mathrm{ANO} 1$ and $\mathrm{ANO} 2$ are known to be activated by $\mathrm{Ca}^{2+}[5,25]$. Thus, we aligned the $\sim 45$ amino acids flanking the EF-hand like regions of all ANO homologs. As shown in Fig. 3A, acidic amino acids in 285-DGDYEGDNVEFND-297 of ANO1 were found to be well conserved in ANO2, but not in the other ANO homologs. This coincidence of homology and $\mathrm{Ca}^{2+}$ response also suggests that the EF-hand loop-like region is involved in the modulation for the $\mathrm{Ca}^{2+}$ sensitivity of $\mathrm{ANO} 1$ and $\mathrm{ANO} 2$.

ANO2 was found to be activated by $\left[\mathrm{Ca}^{2+}\right]_{\mathrm{i}}$ in a dose-dependent manner (Fig. 3B). ANO2 also showed voltage-dependent $\mathrm{Ca}^{2+}$ sensitivity (Fig. 3C and 3D). At a holding potential of $-80 \mathrm{mV}$, the $\mathrm{EC}_{50}$ for the $\mathrm{Ca}^{2+}$ activation of ANO2 was $12.5 \mu \mathrm{M}$. However, at +80 $\mathrm{mV}$ the $\mathrm{EC}_{50}$ reduced to $2.6 \mu \mathrm{M}$. Therefore, as observed in ANO1, ANO2 also showed a greater sensitivity to $\mathrm{Ca}^{2+}$ at depolarization than at hyperpolarization.

Because single-channel currents of ANO2 mutants were too 


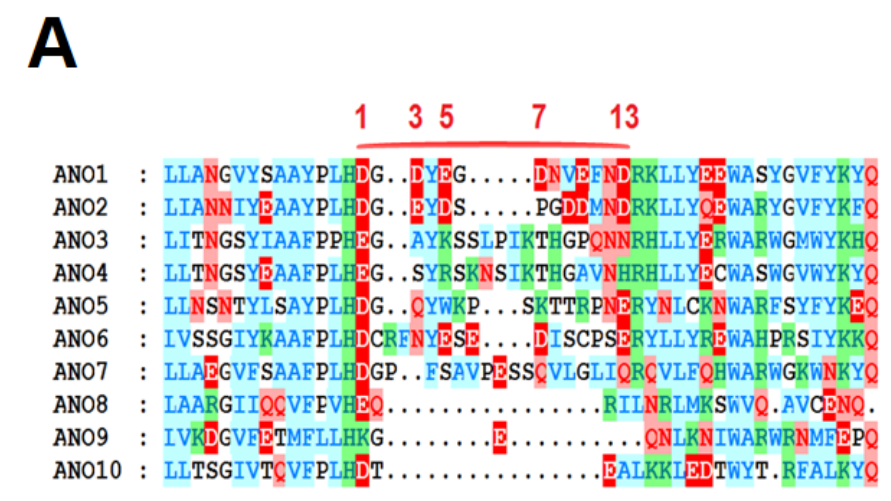

C

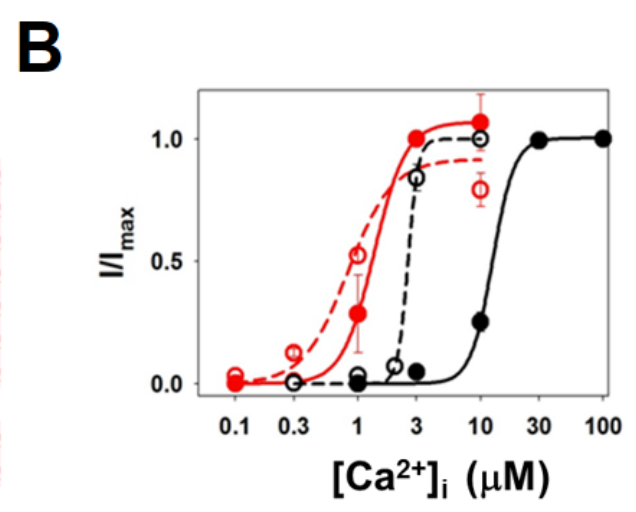

D

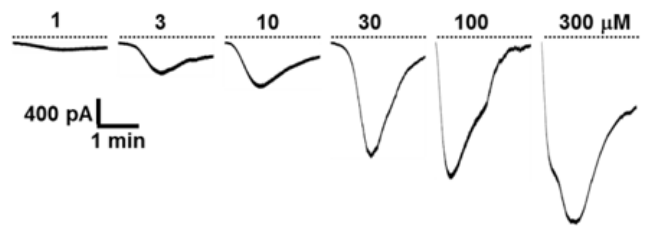

ANO2 D316A/E318A/D320A ( 4 )

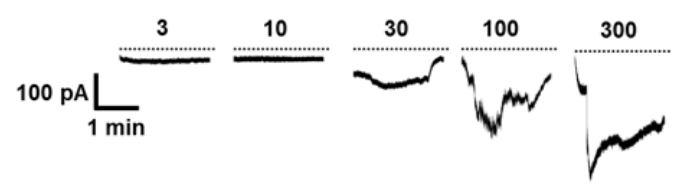

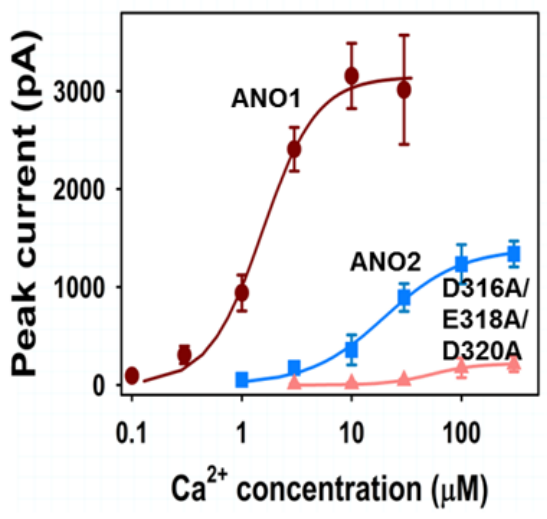

Fig. 3. Mutation of the EF-hand like region of ANO2 confers $\mathrm{Ca}^{2+}$ sensitivity. (A) The sequence alignments of EF-hand like regions in the ANO family. ANO1 and ANO2 have consensus sites in the EF-hand like region. Red; negatively-charged, pink; polar uncharged, green; positively charged, and blue; hydrophobic amino acid side chain. (B) Dose-response relationships of ANO1 (red lines) and ANO2 (black lines) activation by various concentrations of $\mathrm{Ca}^{2+}$ applied to the baths of inside-out patches of ANO1 or ANO2-transfected HEK cells ( $\mathrm{n}=5 \sim 8$ ) at $\mathrm{E}_{\text {hold }}-80 \mathrm{mV}$ (straight line) or $+80 \mathrm{mV}$ (broken line). ANO1 at $+80 \mathrm{mV}, \mathrm{EC}_{50}=0.8 \mu \mathrm{M}\left(\mathrm{n}_{\mathrm{h}}=2.4\right), \mathrm{ANO} 1$ at $-80 \mathrm{mV}, \mathrm{EC}_{50}=1.4 \mu \mathrm{M}\left(\mathrm{n}_{\mathrm{h}}=3.4\right), \mathrm{ANO} 2$ at $+80 \mathrm{mV}, \mathrm{EC}_{50}=2.6 \mu \mathrm{M}\left(\mathrm{n}_{\mathrm{h}}=10.4\right), \mathrm{ANO} 2$ at $-80 \mathrm{mV}$, $\mathrm{EC}_{50}=12.5 \mu \mathrm{M}\left(\mathrm{n}_{\mathrm{h}}=4.9\right)$. (C) Whole-cell currents of ANO2 (upper panel) and of ANO2 D316A/E318A/D320A mutant (lower panel) recorded from HEK cells transfected with ANO2 or its mutant with various $\left[\mathrm{Ca}^{2+}\right]_{\mathrm{i}}$ (from 1 to $300 \mu \mathrm{M}$ ) in the pipette solution. (D) The dose-response characteristics of the whole-cell currents of $\mathrm{ANO} 1(\bullet), \mathrm{ANO} 2(\boldsymbol{\bullet})$, and ANO2 D316A/E318A/D320A mutant $(\boldsymbol{\Delta})(\mathrm{n}=5 \sim 10)$. $\mathrm{E}_{\text {hold }}=-60 \mathrm{mV}$. ANO1 EC $\mathrm{E}_{50}=1.6 \mu \mathrm{M}, \mathrm{ANO}_{2}$ $\mathrm{EC}_{50}=20.0 \mu \mathrm{M}, \mathrm{ANO} 2 \mathrm{D} 316 \mathrm{~A} / \mathrm{E} 318 \mathrm{~A} / \mathrm{D} 320 \mathrm{~A}$ mutant $\mathrm{EC}_{50}=53.4 \mu \mathrm{M}$.

small to measure even at the maximum $\mathrm{Ca}^{2+}$ concentration used $(300 \mu \mathrm{M})$, whole-cell currents of wild-type ANO2 and of its D316A/E318A/D320A mutant were used to obtain an approximation of the dose-response relationships of ANO2 mutants $\left(E_{\text {hold }}=-60 \mathrm{mV}\right)$. The overall shape of ANO2 current response to $\mathrm{Ca}^{2+}$ was found to be similar to that of ANO1, except that a higher $\left[\mathrm{Ca}^{2+}\right]_{\mathrm{i}}$ was required for activation. Appreciable currents were activated only by $\mathrm{Ca}^{2+}$ concentrations higher than $3 \mu \mathrm{M}$. The $\mathrm{EC}_{50}$ of $\mathrm{ANO} 2$ was found to be $20.0 \mu \mathrm{M}$, which is a 20 -fold greater than that of ANO1 $\left(E_{50}=1.0 \mu \mathrm{M}\right)(\mathrm{Fig} .3 \mathrm{C}$ and 3D). When three acidic amino acids (316-DGEYDSPGDDMND-328) in the EF-hand like region in ANO2 were replaced with Ala (D316A/E318A/D320A),
$\mathrm{Ca}^{2+}$ sensitivity and current density were substantially reduced $\left(\mathrm{EC}_{50}=54.3 \mu \mathrm{M}\right.$ vs $20 \mu \mathrm{M}$ for wild type ANO2) (Fig. 3C and 3D). These acidic amino acids correspond to the 1,3 , and 5 residues of the common EF hand of calmodulin, and are known to be essential for $\mathrm{Ca}^{2+}$ coordination [23]. These results further suggest that the acidic amino acids in the EF-hand like regions of ANO1 and $\mathrm{ANO} 2$ are an important modulatory residue in $\mathrm{Ca}^{2+}$-induced ANO1 activation.

\section{Structural properties of ANO1 from molecular modeling analysis}

To ascertain the 3D structure-based involvement of EF-hand like 


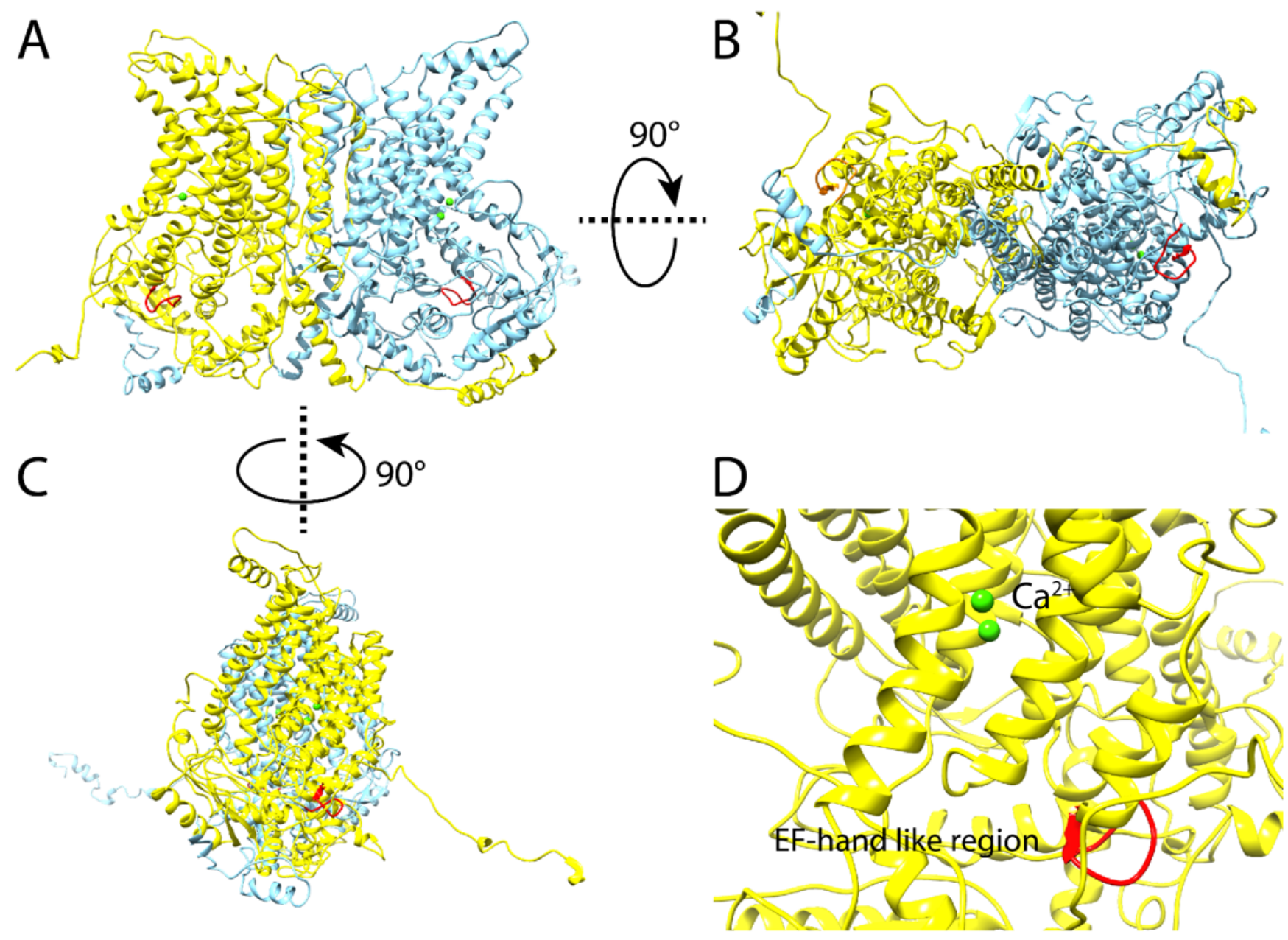

Fig. 4. Three-dimensional structures of ANO1. (A) The secondary structure of ANO1 was shown with ribbon presentation method, colored by yellow (left subunit of dimer), cyan (right subunit of dimer), green ( $\mathrm{Ca}^{2+}$ ions), and red (EF-hand like region, 285 297 residue numbers), respectively. (B) View of dimer structure of ANO1 from extracellular side. (C) View of structure from the membrane looking towards EF-hand like region (colored by red) and $\mathrm{Ca}^{2+}$ binding site (shown with greed colored $\mathrm{Ca}^{2+}$ ions), respectively. (D) Close-up view of EF-hand like region and $\mathrm{Ca}^{2+}$ binding site.
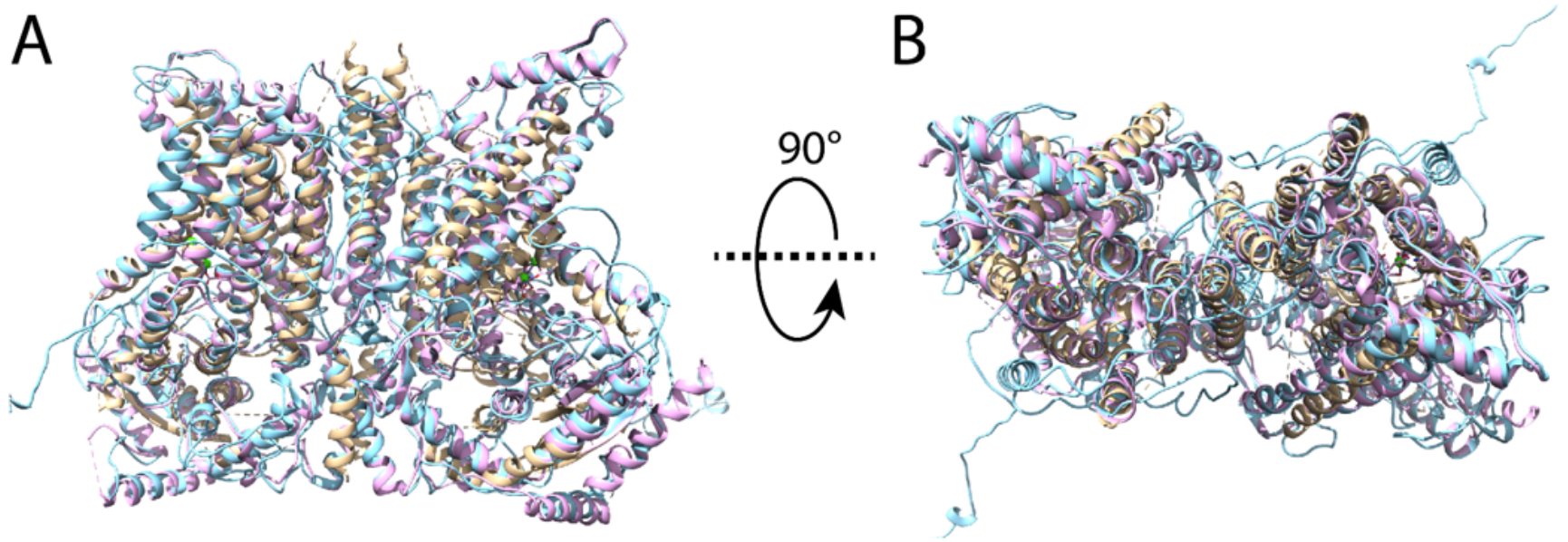

Fig. 5. Comparison of three-dimensional structures of ANO1. (A) Modeled structure of mANO1 (cyan colored), structure from cryoEM (gold colored), and structure from x-ray crystallography (magenta colored) were superimposed using UCSF Chimera Match Maker module. (B) View of structures of ANO1 from extracellular side. 


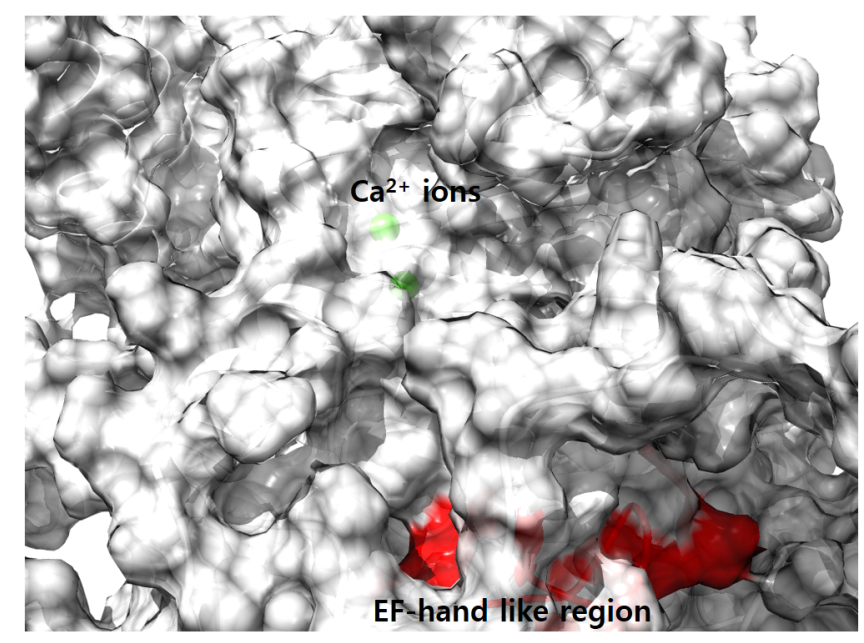

Fig. 6. Surface model of mANO1. The EF-hand like region and $\mathrm{Ca}^{2+}$ ions were colored by red and green, respectively.

region in $\mathrm{Ca}^{2+}$ binding to ANO1, we performed molecular modeling analysis using various in silico methods such as I-TASSER, ROSETTA [19], RaptorX [26], Phyre2 [27]. All modeled structures were validated using PSVS to select reasonable structure among predicted structures, and several modeled structures from I-TASSER algorithm was selected for further refinement. Finally, the best one structure among modeled structures was selected based on energy-scoring function and validation results. This model shows that the overall folding of ANO1 structure was similar with that of nhTMEM16 structure used as template during homology modeling (Fig. 4).

The three-dimensional structure of ANO1 shows a similar overall shape when compared to the nhTMEM16 structure used for modeling (Fig. 5). ANO1 has bundle of transmembrane helices like nhTMEM16, and specifically, the N-terminal region is longer than nhTMEM16. The long N-terminal region is composed of more than 120 amino acids and is expected to form a flexible loop rather than a transmembrane helix. Before reaching the first TM, several helix forms existed and some helical region seems to be able to bind $\mathrm{Ca}^{2+}$ ion. In modeled structure, the EF-hand like region was located at the entrance leading to the $\mathrm{Ca}^{2+}$ binding site (Fig. 6). Therefore, this EF-hand like region could have effect on the migration of $\mathrm{Ca}^{2+}$ ions between the cytoplasm and the $\mathrm{Ca}^{2+}$ binding site (Fig. 4 and 7).

\section{DISCUSSION}

\section{Activation of ANO1 by $\mathrm{Ca}^{2+}$ and voltage}

As is observed for native CaCCs $[2,4,24]$, the rate of ANO1 activation by depolarization increases with $\left[\mathrm{Ca}^{2+}\right]_{\mathrm{i}}$. In addition,

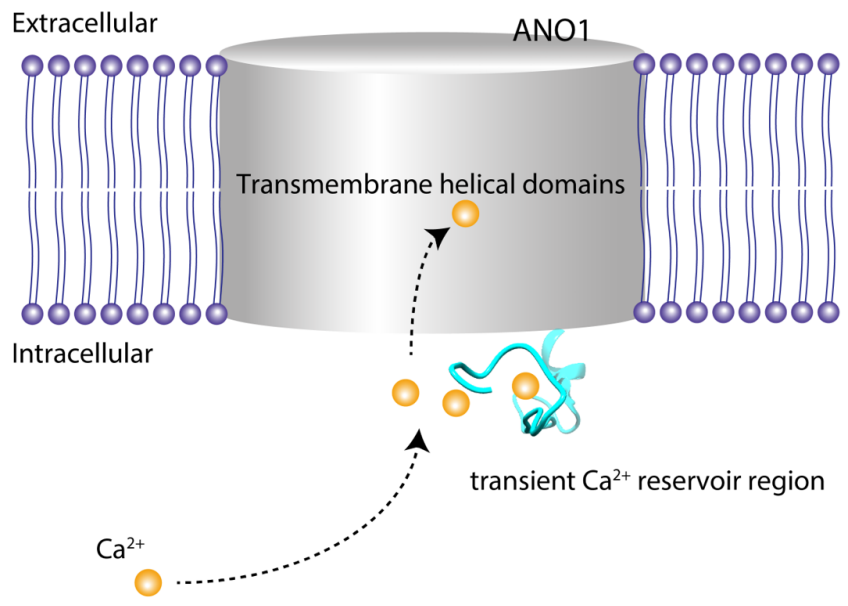

Fig. 7. Schematic representation of migration of $\mathrm{Ca}^{2+}$ ions mediated by transient $\mathrm{Ca}^{2+}$ reservoir region.

the deactivation of ANO1 was also found to be $\mathrm{Ca}^{2+}$ and voltage dependent. Kuruma et al. [2] derived a model for the $\mathrm{Ca}^{2+}$ - and voltage-dependent activation and deactivation mechanisms of native CaCCs, which fits well with actual current responses to $\mathrm{Ca}^{2+}$ and voltage. This model contains three main features. First, the activations of CaCCs depend mainly on $\mathrm{Ca}^{2+}$, and not on voltage. Second, more than one $\mathrm{Ca}^{2+}$ ion is required to activate CaCCs, and third, deactivation depends on voltage. Evidently, ANO1 appears to possess all three of these features of endogenous CaCCs. First, ANO1 was easily activated by $\mathrm{Ca}^{2+}$ but rarely activated by voltage at low $\left[\mathrm{Ca}^{2+}\right]_{i}$ (Fig. 2A). Second, the Hill coefficient of $\mathrm{Ca}^{2+}$ for the activation of wild-type ANO1 was 3.7, and this reduced to less than 1 when Glu and Asp residues in the EF-hand like region were replaced by Ala. These results suggest that the binding of more than one $\mathrm{Ca}^{2+}$ is required for ANO1 activation and that acidic amino acids in the EF-hand like region cooperatively collect and transport $\mathrm{Ca}^{2+}$. Third, the deactivation of ANO1 is voltage dependent, because hyperpolarization was found to deactivate ANO1 even in the presence of $\mathrm{Ca}^{2+}$ (Fig. 2). Thus, the mechanisms underlying the activation and deactivation of $\mathrm{ANO} 1 \mathrm{by}^{2+}$ and voltage appear to be similar to those proposed for native CaCCs.

\section{Mechanism of $\mathrm{Ca}^{2+}$ binding and subsequent ion conduction of $M A N O 1$}

The $\mathrm{Ca}^{2+}$ binding of sites of ANO1 are well characterized in detail in cryo-EM studies [13,28]. According to these studies, Clconducting pores are present in each subunit, which are formed by transmembrane helices $\alpha 3-\alpha 7$. The pore has a narrow neck that determines $\mathrm{Cl}$ - conductance and wide vestibule $[13,28]$. When $\mathrm{Ca}^{2+}$ binds four acidic amino acids in $\alpha 7$ and $\alpha 8$, the positive charge density in $\alpha 7$ and $\alpha 8$ attracts the E654 residue in $a 6$, which makes 
a comparably large movement of $a 6$. This conformational change of $a 6$ induces the opening of the neck of the pore, thus conducting $\mathrm{Cl}^{-}$[13]. Mammalian ANO1 has longer cytoplasmic N-terminal extension than nhTMEM16, whose function is not well characterized. The long region of $\mathrm{N}$-terminal is located adjacent to the membrane surface rather than the region passing through the membrane. We do not know how the EF-hand like region in the $\mathrm{N}$-terminus links to the $\mathrm{Ca}^{2+}$ binding sites in $\alpha 6, \alpha 7$, or $\alpha 8$. The strong phenotype of mutants in this region clearly suggests its role in modulating $\mathrm{Ca}^{2+}$-dependent activation of ANO1. Perhaps, the EF-hand like region acts like a transient $\mathrm{Ca}^{2+}$ reservoir, which could help the $\mathrm{Ca}^{2+}$ ions move to the $\mathrm{Ca}^{2+}$ binding site embedded inside the lipid bilayer (Fig. 7).

\section{The EF-hand like region controls voltage dependence of ANO1}

One of the canonical properties of CaCCs is its activation by $\mathrm{Ca}^{2+}$ and voltage [2]. As a CaCC, ANO1 is also activated by voltage and $\mathrm{Ca}^{2+}[5,7,9,29]$. The activation of ANO1 by voltage requires intracellular $\mathrm{Ca}^{2+}$ because without $\mathrm{Ca}^{2+}$, depolarization fails to open ANO1 $[9,29]$. Thus, it seems the voltage- and $\mathrm{Ca}^{2+}$-dependent gating of ANO1 is tightly coupled. A few sites were suggested to be essential for the voltage- and $\mathrm{Ca}^{2+}$-dependent activation of ANO1. Structural analysis combined to mutagenesis study suggests that the sixth TM is essential for Ca2+ as well as voltage-dependent gating of mouse ANO1 [30]. A highly charged segment in the first intracellular loop (444-EEEEEAVK-451), an intracellular loop between TM $\alpha 2$ and TM $\alpha 3$, is important for $\mathrm{Ca}^{2+}$ - and voltagedependent gating $[9,29]$. Deletion or mutation of EAVK residues profoundly reduced $\mathrm{Ca}^{2+}$ and voltage-activated currents suggesting the $\mathrm{Ca}^{2+}$ and voltage sensitivities are also coupled [9, 29]. The EF-hand like region in ANO1 appears to control the voltage dependence of ANO1. When we replaced four acidic amino acids in the EF-hand like region with Ala (the AGAYAGA mutant), the slow activation of ANO1 by voltage at low $\mathrm{Ca}^{2+}$, voltage-dependent conductance change, and voltage dependent increases in $\tau_{d}$ were largely eliminated (Fig. 2), which strongly suggest that the EFhand like region also controls the voltage dependence of ANO1. As the voltage dependency relies on the binding of $\mathrm{Ca}^{2+}$ to the $\mathrm{Ca}^{2+}$ binding sites in the TM $a 6$ whose conformational change gates the channel, it is conceivable that the ionic interaction between $\mathrm{Ca}^{2+}$ and oxygen atoms of carboxyl or carbonyl groups of the acidic amino acids in the EF-hand like region function as a voltage sensor. Voltage change in this region may affect the ionic interaction between $\mathrm{Ca}^{2+}$ and carboxyl or carbonyl groups in the region. Alternatively, the acidic amino acids in the EF-hand like region may interact with voltage sensors in the pore region or other areas as shown in the voltage-gated proton channel, Hv1 [31].

\section{Structural element of the EF-hand like region of ANO1}

EF-hand like regions in $\mathrm{Ca}^{2+}$ sensors and buffers usually has common structural features. In particular, canonical EF-hand regions commonly contain Asp and Glu residues in their 1, 3, 5, 9, and 12 positions for $\mathrm{Ca}^{2+}$ coordination [22, 23]. Furthermore, EF-hand proteins have a helix-loop-helix structure, and the helix-loop-helix motifs are present in pairs. In the present study, we performed computer modeling to determine the 3-dimensional structure of the EF-hand like region of ANO1. However, this region of ANO1 does not share similarity with the 3-dimensional structure of the EF hand of calmodulin, largely because helices flanking the loop region are lacking. Furthermore, the EF-hand like region in ANO1 is not present in pairs. Thus, this region in ANO1 does not fall into the canonical EF hand category. However, the primary structure of the ANO1 EF-hand like region is closely related to that of the EF hand because acidic amino acids are aligned well with those of the canonical EF-hand like region. For example, in canonical EF hands, Asp and Glu residues at the 1, 3, 5, 9, and 12 positions provide side-chain carboxylic oxygens for $\mathrm{Ca}^{2+}$ coordination [22, 23]. In addition, the backbone carbonyl oxygen at position 7 is also essential for $\mathrm{Ca}^{2+}$ chelation. Likewise, in the EF-hand like region of ANO1, Asp and Glu residues are present at the 1,3,5, 10, and 13 positions, which also presumably provide side-chain carboxylic oxygens for $\mathrm{Ca}^{2+}$ coordination. In addition, Glu at position 7 also provides a side-chain carboxylic oxygen for $\mathrm{Ca}^{2+}$ coordination. Thus, the structural element in this region of ANO1 explains why this region is essential for the activation by $\mathrm{Ca}^{2++}$ (page 20 21).

\section{ACKNOWLEDGEMENTS}

This research was supported by a grant from the National Research Foundation (NRF) of Korea (NRF-2018R1D1A1B07049840) and the National Research Foundation of Korea, funded by the Ministry of Science and ICT (2011-0018358). Also, this work was partly supported by the GRRC program of Gyeonggi province (GRRC-CHA2017-A02, Validity and Safety Evaluation of Regional Specialized Resources).

\section{REFERENCES}

1. Hartzell C, Putzier I, Arreola J (2005) Calcium-activated chloride channels. Annu Rev Physiol 67:719-758.

2. Kuruma A, Hartzell HC (2000) Bimodal control of a Ca(2+)activated $\mathrm{Cl}(-)$ channel by different $\mathrm{Ca}(2+)$ signals. J Gen Physiol 115:59-80. 
3. Arreola J, Melvin JE, Begenisich T (1996) Activation of calcium-dependent chloride channels in rat parotid acinar cells. J Gen Physiol 108:35-47.

4. Nilius B, Prenen J, Voets T, Van den Bremt K, Eggermont J, Droogmans G (1997) Kinetic and pharmacological properties of the calcium-activated chloride-current in macrovascular endothelial cells. Cell Calcium 22:53-63.

5. Yang YD, Cho H, Koo JY, Tak MH, Cho Y, Shim WS, Park SP, Lee J, Lee B, Kim BM, Raouf R, Shin YK, Oh U (2008) TMEM16A confers receptor-activated calcium-dependent chloride conductance. Nature 455:1210-1215.

6. Caputo A, Caci E, Ferrera L, Pedemonte N, Barsanti C, Sondo E, Pfeffer U, Ravazzolo R, Zegarra-Moran O, Galietta LJ (2008) TMEM16A, a membrane protein associated with calciumdependent chloride channel activity. Science 322:590-594.

7. Schroeder BC, Cheng T, Jan YN, Jan LY (2008) Expression cloning of TMEM16A as a calcium-activated chloride channel subunit. Cell 134:1019-1029.

8. Ferrera L, Caputo A, Ubby I, Bussani E, Zegarra-Moran O, Ravazzolo R, Pagani F, Galietta LJ (2009) Regulation of TMEM16A chloride channel properties by alternative splicing. J Biol Chem 284:33360-33368.

9. Xiao Q, Yu K, Perez-Cornejo P, Cui Y, Arreola J, Hartzell HC (2011) Voltage- and calcium-dependent gating of TMEM16A/Anol chloride channels are physically coupled by the first intracellular loop. Proc Natl Acad Sci U S A 108:88918896.

10. Lee J, Jung J, Tak MH, Wee J, Lee B, Jang Y, Chun H, Yang DJ, Yang YD, Park SH, Han BW, Hyun S, Yu J, Cho H, Hartzell $\mathrm{HC}$, Oh U (2015) Two helices in the third intracellular loop determine anoctamin 1 (TMEM16A) activation by calcium. Pflugers Arch 467:1677-1687.

11. Tien J, Peters CJ, Wong XM, Cheng T, Jan YN, Jan LY, Yang $\mathrm{H}$ (2014) A comprehensive search for calcium binding sites critical for TMEM16A calcium-activated chloride channel activity. Elife 3:e02772.

12. Brunner JD, Lim NK, Schenck S, Duerst A, Dutzler R (2014) $\mathrm{X}$-ray structure of a calcium-activated TMEM16 lipid scramblase. Nature 516:207-212.

13. Paulino C, Neldner Y, Lam AK, Kalienkova V, Brunner JD, Schenck S, Dutzler R (2017) Structural basis for anion conduction in the calcium-activated chloride channel TMEM16A. Elife 6:e26232.

14. Zhang Y (2008) I-TASSER server for protein 3D structure prediction. BMC Bioinformatics 9:40.

15. Pearlman DA, Case DA, Caldwell JW, Ross WS, Cheatham III TE, DeBolt S, Ferguson D, Seibel G, Kollman P (1995) AM-
BER, a package of computer programs for applying molecular mechanics, normal mode analysis, molecular dynamics and free energy calculations to simulate the structural and energetic properties of molecules. Computer Physics Communications 91:1-41.

16. Case DA, Cheatham TE 3rd, Darden T, Gohlke H, Luo R, Merz KM Jr, Onufriev A, Simmerling C, Wang B, Woods RJ (2005) The Amber biomolecular simulation programs. J Comput Chem 26:1668-1688.

17. Pettersen EF, Goddard TD, Huang CC, Couch GS, Greenblatt DM, Meng EC, Ferrin TE (2004) UCSF Chimera--a visualization system for exploratory research and analysis. J Comput Chem 25:1605-1612.

18. Meng EC, Pettersen EF, Couch GS, Huang CC, Ferrin TE (2006) Tools for integrated sequence-structure analysis with UCSF Chimera. BMC Bioinformatics 7:339.

19. Bonneau R, Tsai J, Ruczinski I, Chivian D, Rohl C, Strauss CE, Baker D (2001) Rosetta in CASP4: progress in ab initio protein structure prediction. Proteins Suppl 5:119-126.

20. Bhattacharya A, Tejero R, Montelione GT (2007) Evaluating protein structures determined by structural genomics consortia. Proteins 66:778-795.

21. Konc J, Janežič D (2017) ProBiS tools (algorithm, database, and web servers) for predicting and modeling of biologically interesting proteins. Prog Biophys Mol Biol 128:24-32.

22. Grabarek Z (2006) Structural basis for diversity of the EFhand calcium-binding proteins. J Mol Biol 359:509-525.

23. Gifford JL, Walsh MP, Vogel HJ (2007) Structures and metalion-binding properties of the Ca2+-binding helix-loop-helix EF-hand motifs. Biochem J 405:199-221.

24. Evans MG, Marty A (1986) Calcium-dependent chloride currents in isolated cells from rat lacrimal glands. J Physiol 378:437-460.

25. Ha GE, Cheong E (2017) Spike frequency adaptation in neurons of the central nervous system. Exp Neurobiol 26:179185.

26. Wang S, Li W, Liu S, Xu J (2016) RaptorX-Property: a web server for protein structure property prediction. Nucleic Acids Res 44:W430-W435.

27. Kelley LA, Mezulis S, Yates CM, Wass MN, Sternberg MJ (2015) The Phyre2 web portal for protein modeling, prediction and analysis. Nat Protoc 10:845-858.

28. Dang S, Feng S, Tien J, Peters CJ, Bulkley D, Lolicato M, Zhao J, Zuberbühler K, Ye W, Qi L, Chen T, Craik CS, Jan YN, Minor DL Jr, Cheng Y, Jan LY (2017) Cryo-EM structures of the TMEM16A calcium-activated chloride channel. Nature 552:426-429. 
29. Xiao Q, Cui Y (2014) Acidic amino acids in the first intracellular loop contribute to voltage- and calcium- dependent gating of anoctamin1/TMEM16A. PLoS One 9(6): e99376.

30. Peters CJ, Gilchrist JM, Tien J, Bethel NP, Qi L, Chen T, Wang L, Jan YN, Grabe M, Jan LY (2018) The sixth transmembrane segment is a major gating component of the TMEM16A calcium-activated chloride channel. Neuron 97:1063-1077. e4.

31. DeCoursey TE (2018) Voltage and $\mathrm{pH}$ sensing by the voltagegated proton channel, HV1. J R Soc Interface 15:20180108. 